\title{
Quantitative comparison of immunohistochemical staining intensity in tissues fixed in formalin and Histochoice
}

\author{
D. Geoffrey Vince ${ }^{\mathrm{a}, *}$, Abdelghani Tbakhi ${ }^{\mathrm{b}}$, Ajeetkumar Gaddipati ${ }^{\mathrm{a}, \mathrm{c}}$, Robert M. Cothren ${ }^{\mathrm{a}}$, \\ J. Fredrick Cornhill ${ }^{\mathrm{a}}$ and Raymond R. Tubbs ${ }^{\mathrm{b}}$ \\ ${ }^{a}$ Whitaker Biomedical Imaging Laboratory, Department of Biomedical Engineering, \\ ${ }^{\mathrm{b}}$ Department of Clinical Pathology, The Cleveland Clinic Foundation, Cleveland, Ohio \\ ${ }^{\mathrm{c}}$ Biomedical Engineering Center, The Ohio State University, Columbus, Ohio
}

Received 10 March 1997

Revised 7 June 1997

Accepted 10 June 1997

\begin{abstract}
Formaldehyde fixatives have traditionally been used to preserve tissues as they impart excellent morphological preservation. Formaldehyde fixes tissue by cross linking, a process which can reduce the antigenicity of tissue and weakens the intensity of immunohistochemical stains. Preliminary studies have shown that Histochoice tissue fixative offers equal or greater staining intensity than neutral buffered formalin (NBF). This study compares these fixatives quantitatively and presents the results in unambiguous statistical terms.

Tissue samples were collected, bisected, and fixed in NBF or Histochoice. The sections were stained with a total of 21 antibodies, and color images were collected. The hue, saturation, and value were determined for each positive pixel and an ANOVA performed. Small differences in hue were noted in 8 of 21 cases. Histochoice-fixed tissue gave a greater mean saturation than NBF with $57.1 \%$ of the antibodies tested. No significant difference in the saturation was detected in $28.6 \%$ of the cases; NBF gave higher mean saturation levels with only $14.3 \%$ of the antibodies. Histochoice-fixed tissue was found to give lower values in $66.7 \%$ of cases than those prepared with NBF, indicating darker staining. These results show that Histochoice produces staining intensity that is comparable, and in many cases superior, to formalin.
\end{abstract}

Keywords: Image analysis, fixation, immunohistochemistry, color analysis

\section{Introduction}

Neutral buffered formalin (NBF) has traditionally been used as a tissue fixative because it imparts excellent morphological preservation, penetrates quickly, and facilitates sectioning by hardening the tissue [15]. Unfortunately, formalin-based products fix tissue by crosslinking protein molecules, a process which can reduce immunohistochemical staining intensity [16]. Several digestion/antigen retrieval systems have recently become available through commercial sources that work by breaking

${ }^{*}$ Corresponding author: D. Geoffrey Vince, Department of Biomedical Engineering, Mail Drop Wb3, The Cleveland Clinic Foundation, 9500 Euclid Avenue, Cleveland, OH 44195, USA. Tel.: +1 216444 1211; Fax: +1 2164449198 ; Email: vince@bme.ri.ccf.org. 
the crosslinks formed by formalin fixation. Most systems rely on heating heavy metal salts or acid solutions and may cause mild to moderate tissue damage, thus compromising morphometric analysis. Previous studies have also identified formaldehyde, a major component of formalin, as a mutagen and carcinogen [4,9]. Even at relatively low levels, formaldehyde has been shown to cause allergic reactions and extreme skin irritation in some individuals [14]. In addition, formalin is becoming increasingly expensive to use because of more stringent laboratory safety procedures.

Numerous non formalin fixatives have been used in previous immunohistochemical studies, although many of these use OSHA-regulated heavy metal salts, such as mercury, to denature macromolecules within the tissue [15]. A number of proprietary fixatives are commercially available, and although information on their mechanism of action is scarce, they have been used successfully by several investigators $[6,18]$.

Preliminary qualitative studies in the authors' laboratories have shown that the proprietary fixative Histochoice (Amresco, Solon, OH, USA) offers a staining intensity equal to, and in many cases greater than, that of NBF, the current "gold-standard" in tissue fixation. The primary active ingredient in Histochoice is ethanedial, which acts in a similar manner to formalin, in that it forms crosslinks in the tissue. One important difference is that the crosslinks formed by ethanedial are much weaker than those formed by formaldehyde and can be easily broken. However, no automated, quantitative assessment of staining intensity has been performed. In this study, we used a custom-engineered, automated image analysis system to assess the immunohistochemical staining intensity of tissues fixed in NBF and Histochoice, and present the results in unambiguous statistical terms.

\section{Methods}

\subsection{Sample collection and preparation}

Tissue samples from seven patients, comprising normal tonsil $(n=3)$, colon carcinoma $(n=1)$, prostate carcinoma $(n=2)$, and normal pancreas $(n=1)$, were obtained from the Department of Anatomic Pathology at The Cleveland Clinic Foundation. Each piece of tissue was bisected and fixed for eight hours, half in 10\% NBF and half in Histochoice. The tissue was then processed and paraffin embedded in an identical manner according to standard laboratory procedures. Sections were cut from each block at $4 \mu \mathrm{m}$, collected onto electrostatically charged slides, and baked at $60^{\circ} \mathrm{C}$ for $60 \mathrm{~min}$. Two sections were collected for each antibody and immunostained as described below.

\subsection{Immunohistochemical staining procedure}

The antibodies used in this study are listed in Table 1 . The paraffin was removed from the baked tissue sections in three changes of xylene, and the tissue hydrated through graded alcohols before rinsing in phosphate buffered saline (PBS).

Immunohistochemical staining was performed using a Jung Histostainer (Leica, IL, USA), with processing occurring at $30^{\circ} \mathrm{C}$. The first stage of the procedure involved the application of $1 \%$ hydrogen peroxide solution in methanol for $5 \mathrm{~min}$ to remove any endogenous peroxidase present in the tissue section. For mouse monoclonal primary antibodies, a blocking solution comprising a $1: 10$ dilution of normal rabbit serum (Dako, CA, USA) in PBS was then added for $10 \mathrm{~min}$ before application of the primary antibody. The required dilutions of antibody were prepared using $1 \%$ bovine serum albumin (BSA) in PBS. Incubation occurred at $30^{\circ} \mathrm{C}$ for $60 \mathrm{~min}$, and a $1: 200$ dilution of biotinylated 
Table 1

Qualitative assessment of staining intensity for samples of human tonsil, colon, prostate, and pancreas fixed in either Histochoice or NBF

\begin{tabular}{lllrcc}
\hline Tissue & Antibody & Supplier & Protein conc. & \multicolumn{2}{c}{ Grade } \\
\cline { 5 - 6 } & & & & Reviewer 1 & Reviewer 2 \\
\hline Tonsil 3-patients & AE1/3 & Hybertech & $1 \mathrm{mg} / \mathrm{ml}$ & $\mathrm{S}$ & $\mathrm{S}$ \\
& BLA & BioGenex & $5.4 \mathrm{mg} / \mathrm{ml}$ & $\mathrm{S}$ & $\mathrm{S}$ \\
& CAM5.2 & B\&D & $25 \mu \mathrm{g} / \mathrm{ml}$ & $\mathrm{S}$ & $\mathrm{F}$ \\
& CD15 & B\&D & $50 \mu \mathrm{g} / \mathrm{ml}$ & $\mathrm{H}$ & $\mathrm{H}$ \\
& CD20 & Dako & $7.5 \mathrm{mg} / \mathrm{ml}$ & $\mathrm{F}$ & $\mathrm{S}$ \\
& CD57 & B\&D & $12.5 \mu \mathrm{g} / \mathrm{ml}$ & $\mathrm{S}$ & $\mathrm{H}$ \\
& CD64 & Dako & $19 \mathrm{mg} / \mathrm{ml}$ & $\mathrm{S}$ & $\mathrm{S}$ \\
& CD68 & Dako & $19 \mathrm{mg} / \mathrm{ml}$ & $\mathrm{H}$ & $\mathrm{S}$ \\
& EMA & Dako & $8.6 \mathrm{mg} / \mathrm{ml}$ & $\mathrm{S}$ & $\mathrm{H}$ \\
& Factor VIII* & Dako & $14.5 \mathrm{mg} / \mathrm{ml}$ & $\mathrm{H}$ & $\mathrm{S}$ \\
& kappa* & Dako & $15.1 \mathrm{mg} / \mathrm{ml}$ & $\mathrm{S}$ & $\mathrm{S}$ \\
& lambda* & Dako & $21.5 \mathrm{mg} / \mathrm{ml}$ & $\mathrm{H}$ & $\mathrm{S}$ \\
Polon 1-patient & muramidase* & Dako & $7.1 \mathrm{mg} / \mathrm{ml}$ & $\mathrm{F}$ & $\mathrm{F}$ \\
& S-100* & Dako & $4.5 \mathrm{mg} / \mathrm{ml}$ & $\mathrm{F}$ & $\mathrm{F}$ \\
Pancreas 1-patient & CEA & Dako & $0.2 \mathrm{mg} / \mathrm{ml}$ & $\mathrm{H}$ & $\mathrm{H}$ \\
& PSA* & Dako & $3.2 \mathrm{mg} / \mathrm{ml}$ & $\mathrm{S}$ & $\mathrm{S}$ \\
& PAP* & Dako & $12.9 \mathrm{mg} / \mathrm{ml}$ & $\mathrm{S}$ & $\mathrm{S}$ \\
& Glucagon $^{*}$ & Dako & $0.2 \mathrm{mg} / \mathrm{ml}$ & $\mathrm{H}$ & $\mathrm{S}$ \\
& Chromogranin & Dako & $0.1 \mathrm{mg} / \mathrm{ml}$ & $\mathrm{H}$ & $\mathrm{F}$ \\
& Somatostatin* & $14.5 \mathrm{mg} / \mathrm{ml}$ & $\mathrm{H}$ & $\mathrm{H}$ \\
& Dako & $30 \mathrm{mg} / \mathrm{ml}$ & $\mathrm{H}$ & $\mathrm{H}$ \\
\hline
\end{tabular}

The sections were reviewed and either Histochoice $(\mathrm{H})$ or NBF $(\mathrm{F})$ judged to give the most intense staining. In cases where no difference was observed the sections were determined to be of the same intensity (S). Asterisk (*) indicates polyclonal.

rabbit anti mouse polyclonal antibody (Dako, CA, USA) was added for a further $30 \mathrm{~min}$. The antibody was labeled using an Elite avidin/biotin/peroxidase complex (Vector Laboratories, CA, USA) applied for $30 \mathrm{~min}$. The final stage comprised the addition of 3,3-diaminobenzidine (DAB) as a chromogen (DAB Kit, Vector Laboratories, CA, USA). Between each step, the slides were rinsed twice for $2 \mathrm{~min}$ in PBS. For polyclonal antibodies, a similar procedure was followed except that normal swine serum instead of rabbit serum was used as a blocking agent, and biotinylated swine anti-rabbit polyclonal antibody was used as the link. Following staining, the sections were rinsed in distilled water, counterstained with hematoxylin, dehydrated, and cleared in xylene; and a coverslip placed on the section. The negative control sections were treated in an identical manner to the test tissue except that $1 \%$ BSA in PBS was added instead of primary antibody.

\subsection{Qualitative evaluation}

The tissue sections were independently reviewed by two of the authors (AT and DGV), blinded to the fixation method, who graded the intensity of staining as weak, moderate, or intense. These data were reviewed, and either Histochoice or NBF was assessed as giving the most intense stain. In cases in which there was no discernible difference in the intensity of the fixatives, sections were judged as being the same (Table 1).

\subsection{Quantitative evaluation}

The image analysis software used for cell quantification was custom engineered in the Department of Biomedical Engineering at The Cleveland Clinic Foundation specifically for use in this study. Four 
high-resolution color digital images were acquired from each section using an Ektron 1412 line-scan camera attached to an Olympus BH-2 microscope by an operator blinded to the fixation method. Representative areas of the tissue were chosen, avoiding anomalies such as the edge of the tissue. The use of a $10 \times$ objective and a $2.5 \times$ projection lens produced a pixel size of $0.27 \mu \mathrm{m}$ in an image up to $4000 \times 4000$ pixels. The total area analyzed for each section was $4.8 \mathrm{~mm}^{2}$. The microscope objective used in this study was of S-Plan Apochromatic grade with a high numerical aperture. A total of 408 images were collected in the course of this study.

The color image captured from the high resolution scanner was corrected for non uniform illumination and color distortion using a white field image captured at the same scanning period and under the same conditions as those of the histological sections. The corrected images were then transformed from red-green-blue (RGB) to hue-saturation-value (HSV) color space. In this color system, hue represents the color of the pigment (i.e., brown, red, or green), saturation is a measure of the whiteness of color (i.e., brown, beige, or white), and value is a measure of how much light can penetrate the stain. The images were then displayed on an SGI Indigo 2 workstation; and custom-built software was used to display and segment the tissue clusters in the 3D histogram [5]. This segmentation was performed by adjusting the clusters until only the DAB-stained tissues in the microscopic image were detected. The hue, saturation, and value were determined for each positive pixel in each image and an analysis of variance (ANOVA) was performed on the data using an SAS GLM procedure.

\section{Results}

\subsection{Qualitative evaluation}

The morphological preservation of the tissues fixed in NBF and Histochoice was similar, with no shrinkage or artifact evident. The qualitative assessments show good inter-reviewer agreement, although, as with all subjective evaluations, there was some disagreement in grading staining intensity in some sections, as outlined below. Evaluating the staining intensity for each antibody, the reviewers found that Histochoice-fixed tissue stained more intensely than NBF fixed samples in $19.0 \%$ of cases (Figs 1 and 2) and with equal intensity in $28.6 \%$ of cases (Fig. 3). In 9.5\% of the antigens studied, NBF was judged to produce a more intense stain than Histochoice (Fig. 4). In six cases (28.6\%), the reviewers could not reach consensus on whether Histochoice gave a more intense or similar stain to NBF. In two cases $(9.5 \%)$, there was no agreement on whether NBF produced a more intense or similar intensity stain than Histochoice. In one case $(4.7 \%)$ the reviewers could not agree whether Histochoice or NBF produced the most intense staining (Table 1). Neither manual cell counting nor tissue grading $(0-4)$ were performed due to the highly subjective nature of this assessment. Qualitative evaluation of the slides demonstrated typical staining patterns for each tissue type. Of particular interest was the positive staining of pancreatic acinar cells for chromogranin. This was evident on both the Histochoice and NBF fixed tissue (Fig. 1).

Both reviewers observed slightly higher background staining with the Histochoice-fixed tissue.

\subsection{Quantitative evaluation}

Table 2 demonstrates the mean hue for each of the antigens analyzed. Small differences in hue were noted in 8 of the 21 cases, although these were less than $7 \%$ indicating a very small change in color had occurred. This color change could not be detected by the reviewers. 
(A)

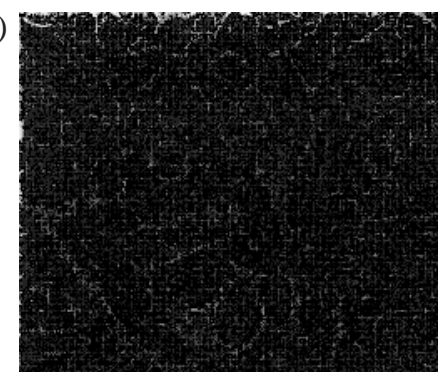

(B)

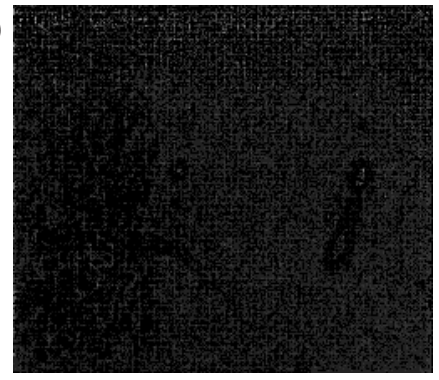

Fig. 1. Section of pancreas stained for chromogranin. Tissue was fixed in (A) Histochoice or (B) NBF (DAB chromogen, $100 \times$ ). Histochoice produced a more intense stain. Note staining of the acinar cells with both fixatives.

(A)

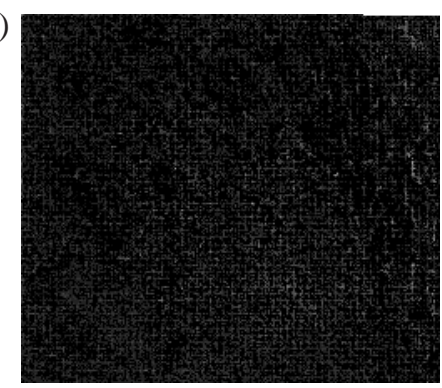

(B)

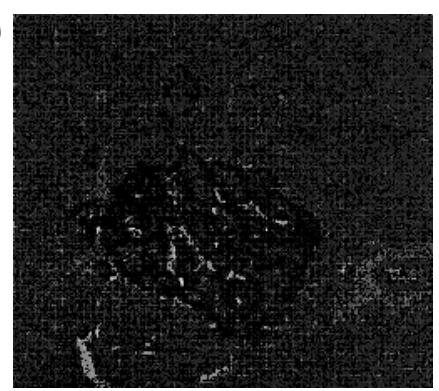

Fig. 2. Human tonsil stained for Factor VIII. Tissue was fixed in (A) Histochoice or (B) NBF (DAB chromogen, 200×). Histochoice produced a more intense stain.

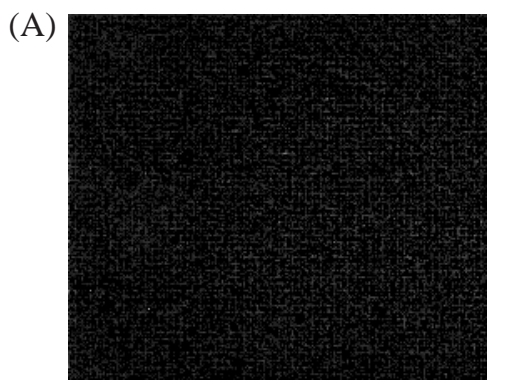

(B)

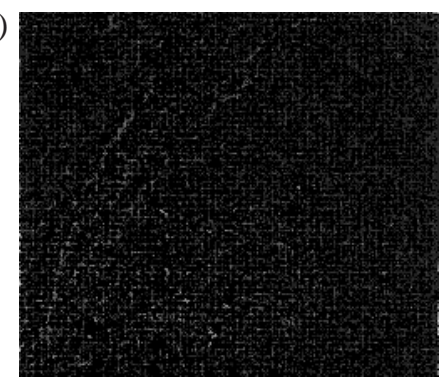

Fig. 3. CD64 staining in human tonsil. Tissue was fixed in (A) Histochoice or (B) NBF (DAB chromogen, 100×). Equal staining intensity produced with Histochoice and NBF.

(A)

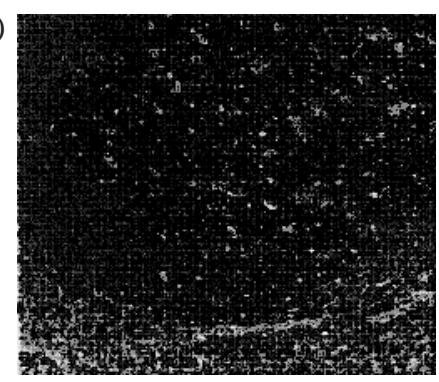

(B)

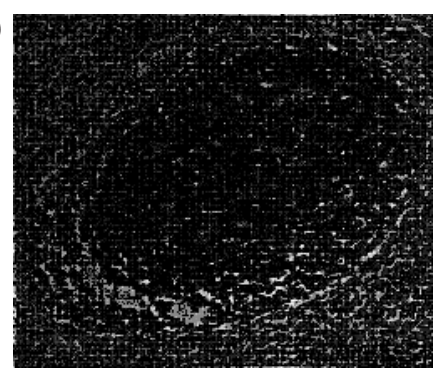

Fig. 4. Section of human tonsil stained for CD20 positive cells. Tissue was fixed in (A) Histochoice or (B) NBF (DAB chromogen, $100 \times)$. NBF produced a more intense stain. 
Table 2

Quantitative analysis of hue for histochoice and formalin fixed tissue

\begin{tabular}{lcccc}
\hline Stain & Histochoice & Neutral buffered formalin & Percentage difference & $p$-value \\
\hline CEA & 270.240 & $\times$ & - & - \\
AE1/3 & 270.436 & 271.336 & 0.3 & $\mathrm{NS}$ \\
BLA & 267.846 & 258.040 & 3.7 & 0.0001 \\
CAM5.2 & 271.971 & 270.920 & 0.4 & $\mathrm{NS}$ \\
CD15 & 257.027 & 259.113 & -0.8 & $\mathrm{NS}$ \\
CD20 & 257.227 & 263.151 & -1.1 & $\mathrm{NS}$ \\
CD57 & 270.782 & 282.478 & -4.3 & 0.0001 \\
CD64 & 278.789 & 271.557 & 2.6 & 0.0001 \\
CD68 & 261.062 & 257.470 & 1.4 & 0.0312 \\
EMA & 269.543 & 268.171 & 0.5 & 0.0023 \\
Factor VIII & 265.493 & 263.548 & 0.7 & $\mathrm{NS}$ \\
kappa & 268.736 & 268.675 & 0.0 & $\mathrm{NS}$ \\
lambda & 270.209 & 269.765 & 0.2 & $\mathrm{NS}$ \\
muramidase & $\times$ & 267.571 & - & - \\
S-100 & 268.249 & 263.778 & 1.7 & 0.0006 \\
PAP & 269.240 & 267.700 & 0.6 & $\mathrm{NS}$ \\
PSA & 266.568 & 265.840 & 0.3 & $\mathrm{NS}$ \\
chromogranin & 265.655 & 249.313 & 6.2 & 0.0002 \\
glucagon & 238.330 & 249.028 & -4.5 & $\mathrm{NS}$ \\
enolase & 252.941 & 250.843 & 0.8 & $\mathrm{NS}$ \\
somatostatin & 250.775 & 239.163 & 4.6 & 0.0065 \\
\hline
\end{tabular}

Table 3

Quantitative analysis of saturation for histochoice and formalin fixed tissue

\begin{tabular}{lcccc}
\hline Stain & Histochoice & Neutral buffered formalin & Percentage difference & $p$-value \\
\hline CEA & 56.256 & $\times$ & - & - \\
AE1/3 & 105.309 & 83.498 & $20.71 \%$ & $\mathrm{NS}$ \\
BLA & 86.661 & 23.823 & $72.51 \%$ & 0.0001 \\
CAM5.2 & 71.534 & 54.169 & $24.28 \%$ & 0.0043 \\
CD15 & 45.118 & 46.238 & $-2.48 \%$ & $\mathrm{NS}$ \\
CD20 & 25.436 & 46.338 & $-82.17 \%$ & 0.0266 \\
CD57 & 62.157 & 44.464 & $28.47 \%$ & 0.0001 \\
CD64 & 45.412 & 43.817 & $3.51 \%$ & $\mathrm{NS}$ \\
CD68 & 53.157 & 35.685 & $32.87 \%$ & 0.0002 \\
EMA & 104.965 & 74.028 & $29.47 \%$ & 0.0001 \\
Factor VIII & 58.375 & 35.546 & $39.11 \%$ & 0.0001 \\
kappa & 68.222 & 85.103 & $-24.74 \%$ & $\mathrm{NS}$ \\
lambda & 90.968 & 62.968 & $30.78 \%$ & 0.0025 \\
muramidase & $\times$ & 66.849 & - & - \\
S-100 & 91.978 & 60.121 & $34.64 \%$ & 0.0001 \\
PAP & 80.895 & 68.335 & $15.53 \%$ & 0.0255 \\
PSA & 105.428 & 95.645 & $9.28 \%$ & $\mathrm{NS}$ \\
chromogranin & 79.843 & 22.525 & $71.79 \%$ & 0.0001 \\
glucagon & 13.458 & 33.513 & $-149.02 \%$ & 0.0113 \\
enolase & 20.060 & 23.409 & $-16.69 \%$ & $\mathrm{NS}$ \\
somatostatin & 17.658 & 10.798 & $38.85 \%$ & 0.0021 \\
\hline NS & & & \\
\hline
\end{tabular}

Histochoice-fixed tissue was found to give higher mean saturation than NBF with twelve $(57.1 \%)$ of the antibodies tested. No significant difference in the saturation was detected with six $(28.6 \%)$ of the antibodies; NBF gave higher mean saturation levels with three (14.3\%) of the antibodies (Table 3). The median mean saturation level was 62.2 for the Histochoice-fixed tissue and 46.2 for the NBF-treated samples. 
Table 4

Quantitative analysis of value for histochoice and formalin fixed tissue

\begin{tabular}{lcccc}
\hline Stain & Histochoice & Neutral buffered formalin & Percentage difference & $p$-value \\
\hline CEA & 178.050 & $\times$ & - & - \\
AE1/3 & 145.476 & 169.698 & $14.27 \%$ & 0.0022 \\
BLA & 149.808 & 88.071 & $-70.10 \%$ & $\mathrm{NS}$ \\
CAM5.2 & 156.951 & 168.888 & $7.07 \%$ & 0.0053 \\
CD15 & 156.501 & 163.920 & $4.53 \%$ & 0.0209 \\
CD20 & 54.743 & 177.106 & $69.09 \%$ & 0.0001 \\
CD57 & 137.469 & 174.694 & $21.31 \%$ & 0.0001 \\
CD64 & 181.466 & 181.088 & $-0.21 \%$ & $\mathrm{NS}$ \\
CD68 & 146.458 & 177.558 & $17.52 \%$ & 0.0001 \\
EMA & 143.428 & 166.004 & $13.60 \%$ & 0.0001 \\
Factor VIII & 167.341 & 194.694 & $14.05 \%$ & 0.0001 \\
kappa & 143.145 & 151.674 & $5.62 \%$ & 0.0228 \\
lambda & 143.956 & 181.758 & $20.80 \%$ & 0.0001 \\
muramidase & $\times$ & 166.644 & - & - \\
S-100 & 129.836 & 159.001 & $18.34 \%$ & 0.0001 \\
PAP & 158.815 & 155.653 & $-2.03 \%$ & $\mathrm{NS}$ \\
PSA & 113.855 & 119.695 & $4.88 \%$ & $\mathrm{NS}$ \\
chromogranin & 144.318 & 188.087 & $23.27 \%$ & 0.0001 \\
glucagon & 198.340 & 176.550 & $-12.34 \%$ & 0.0164 \\
enolase & 196.076 & 187.880 & $-4.36 \%$ & 0.0171 \\
somatostatin & 196.356 & 202.503 & $3.04 \%$ & 0.0018 \\
\hline
\end{tabular}

Table 5

Analysis of variance determining the significance of fixative, stain, and patient variation

\begin{tabular}{llrrrrc}
\hline Parameter & Source & DF & Type III SS & Mean square & $F$-value & $p$-value \\
\hline Saturation & fixation & 1 & 13005.0 & 13005.0 & 33.31 & 0.0001 \\
& stain & 17 & 109902.7 & 6464.9 & 16.56 & 0.0001 \\
& patient & 3 & 2110.1 & 703.3 & 1.80 & 0.1467 \\
\multirow{2}{*}{ Value } & & & & & \\
& fixation & 1 & 31401.3 & 31401.3 & 20.65 & 0.0001 \\
& stain & 17 & 174039.5 & 10237.6 & 6.73 & 0.0001 \\
& patient & 3 & 9720.3 & 3240.1 & 2.13 & 0.0961 \\
\hline
\end{tabular}

$\mathrm{DF}=$ degrees of freedom; Type III SS $=$ partial sum of squares.

Histochoice-fixed tissue was found to give lower values with fourteen $(66.7 \%)$ of the antibodies than those prepared with NBF. NBF gave lower values than Histochoice with three $(14.3 \%)$ of the antibodies. With four (19.0\%) of antigens there was no significant difference in the value of the stain (Table 4).

When all the data were analyzed by ANOVA, Histochoice and NBF were found to give significantly different mean saturation and value with $p$-values of 0.0001 and 0.0001 and $F$-values of 33.3 and 20.6, respectively. This analysis also demonstrated that the mean saturation $(p=$ $0.0001, F=16.56)$ and mean values $(p=0.0001, F=6.73)$ were significantly different for each of the stains. Variation in saturation or value caused by multiple tissue donors was found not to be significant ( $[p=0.1467, F=1.80$, saturation $],[p=0.0961, F=2.13$, value $]$ ) (Table 5). 


\section{Discussion}

Formaldehyde-based fixatives have routinely been used in clinical pathology laboratories as they preserve cell morphology well. However, with the advent of immunohistochemical staining techniques, serious shortcomings have been identified with these reagents, the most limiting being over-fixation, which occurs when tissue is left in the solution for extended periods, allowing cross linking to proceed until all antigenicity is lost. More recently, there has been considerable concern over the carcinogenicity, and related safety issues, associated with formaldehyde [14]. Although the exact mechanism is not well understood, it is speculated that formaldehyde exists almost entirely as methandiol (methylene glycol), which reacts with proteins by binding to side groups containing active hydrogen. The resulting hydroxymethyl groups formed by this reaction undergo condensation reactions with nearby hydrogen groups, producing methylene bridges between polypeptide chains [17]. However, it is now known that antigenicity is lost progressively during the fixation process, and it appears that the methylene bridges mask the antigens in the tissue and so reduce the number of available binding sites [17]. There is also evidence that formalin can form polymers with storage which contribute disproportionally to the masking effect. In addition to reduced staining intensities, many laboratories are now considering switching to non formalin fixatives for economic and safety reasons [2]. A recent study by Northview Pacific Laboratories [13] has demonstrated that when compared to formalin, Histochoice produced a saving of $\$ 22,522$ in direct costs.

Preliminary studies in our laboratory have shown that Histochoice offers significant improvements in staining intensity over NBF and alleviates many of the safety and disposal problems associated with formalin-based fixatives. The primary active ingredient in Histochoice is ethanedial, which acts in a similar manner to formalin, in that it forms crosslinks in the tissue. One important difference is that the crosslinks formed by ethanedial are much weaker than those formed by formaldehyde and can be easily broken. Unlike formaldehyde, ethanedial is neither a known carcinogen nor OSHA regulated and requires no special disposal regimes. In addition to immunohistochemical applications, studies have also demonstrated its suitability for molecular and genetic studies [1,8,11].

Although qualitatively Histochoice appears to provide significant improvement in staining intensity, no quantitative studies have been performed comparing its performance to the current "gold-standard", NBF. The aim of the present study was therefore to compare in a quantitative, objective manner the immunohistochemical staining intensity of tissues fixed in NBF and Histochoice. This comparison was performed with the aid of automated immunostaining instrumentation and a sophisticated, highresolution image capture, processing, and analysis system. The cells were labeled using a panel of marker-specific primary antibodies and detected by an indirect immunoperoxidase technique. All immunoperoxidase staining was performed using an automated staining procedure, which provided a greater level of control over temperature, humidity, reagent volume, and incubation time than would conventional manual immunostaining methods. Although access to such sophisticated equipment may be limited in a clinical laboratory, its use was essential in this study, as all variables, such as temperature and humidity, must be kept constant to accurately assess differences in the two fixatives.

When evaluating new histology reagents or changes in traditional methodologies, it is vitally important to consider the methods of quantification and interpretation of the stained tissue. In relatively simple tasks, such as cell counting, manual techniques have been shown to be time consuming [19] and of questionable accuracy [7] when compared to automated analysis methods. Automated image analysis techniques also allow measurement of parameters that are difficult or impossible to determine accurately using manual methods. These include determination of hue, saturation, and value. 
The qualitative assessment of staining intensity demonstrated that in nine cases $(42.8 \%)$ the reviewers could not agree on which fixative produced the most intense stain. This result highlights the highly subjective nature of the qualitative assessment. Of particular interest was the positive staining of pancreatic acinar cells for chromogranin. This observation has been noted by other groups but does not appear to be predictive of tumor progression $[3,10]$.

The quantitative data provided in this study present the differences in the staining intensity between the two fixatives in unambiguous statistical terms.

When the histological images were transformed to HSV color space and analyzed quantitatively, only small differences were observed in the hue of the stain. This was not a surprising result, in that the fixation process exclusively affects the antigen-antibody binding reaction and will have little affect on either the link, label, or chromogen steps. As color development (i.e., clear to brown reaction product) is the last step in the immunostaining process and is independent of the number of available antigen-antibody binding sites, it was not expected that the fixative would have a great effect on the color of the DAB reaction product. Significant differences were identified in the saturation of the stains between the two fixatives. Saturation is a measure of the purity of color and enables a distinction to be made between a pure brown and a lighter brown. In the context of immunostaining, the saturation depends upon the density of the available antigens. If all the antigens are available for antibody binding, the resulting horseradish peroxidase complexes (HRP) will be in close proximity to each other. As more HRP enzyme is available to participate in the DAB reaction, a richer brown color will result. If some of the antigens are crosslinked to such an extent that they are no longer available for binding, the reaction sites will be further apart and a light brown product will be visible. This process is, of course, dependent upon the total number of antigenic sites available for a particular antibody before fixation. These differences were reflected in the results in that within the Histochoice group, mean saturation varied from 0 to 105.4 and between 0 and 95.6 for NBF.

When the individual antibodies were compared for the two fixatives, significant differences were observed between the two groups. In $85.7 \%$ of cases, Histochoice gave a significantly higher or equal saturation than NBF. In $14.3 \%$ of cases NBF gave a higher mean saturation. These differences were also reflected in the value, with the median mean value for the Histochoice group being 146.5 and 169.7. Value is a measure of how much light can penetrate the stain; more translucent staining will produce a higher value, more opaque staining a lower value. Histochoice-fixed tissue was found to give lower values in $66.7 \%$ of cases than those prepared with NBF; NBF gave lower values in $14.3 \%$ of cases. In $19.0 \%$ of antigens there was no significant difference in the value of the stain. As with the saturation, value is dependent on the number and distribution of antigenic sites. Histochoice contains mild crosslinking agents which do not bind to antigenic sites as aggressively as formaldehyde and so leave these sites available for antibody attachment.

The staining procedure and antibody concentrations used in this study were routinely used in the authors' laboratories and were optimized for formalin-fixed tissue. As Histochoice appears to maintain antigenicity to a greater extent than NBF, the slight increase in background staining may be attributed to too great a concentration of primary antibody. Current studies in our laboratory have shown that the background staining can be decreased by decreasing the concentration of the primary antibody, without affecting positive staining intensity.

The quality of fixation in all the tissue sections was judged to be similar, irrespective of the fixative used. These data conflict with those of MacDonald et al. [12], who found that Histochoice performed poorly in a single-tissue, single-antibody study of six fixatives. One explanation may be that the authors used manual staining methods, which allow little control over temperature, humidity, or reagent 
volume. Although this may represent the clinical setting, these parameters must be controlled to ensure that any difference observed can be attributed to the change in fixative and was not produced by a variation in temperature or reagent volume. In addition, many commercially available image analysis systems use gray-level images and manual thresholding techniques, or a single global thresholding algorithm, thus limiting the analysis steps. Our study utilized fully automated temperature-, humidity-, time-, and volume-controlled immunostaining instruments and a high-resolution, custom-engineered flexible color image analysis system.

Concerns about formalin toxicity and reduction in immunohistochemical staining intensity have highlighted the need for alternative tissue fixation methods. The use of Histochoice fixative overcomes many of the safety concerns associated with formalin and appears to provide a more saturated and intense immunostaining reaction product with many antibodies.

The results of this study show that Histochoice produces comparable, and in many cases superior, staining intensity to formalin without the associated safety concerns.

\section{Acknowledgements}

We gratefully acknowledge Linda Vargo, BS, for her technical expertise with immunostaining, Bradley Skilton, BS, for aiding in tissue procurement, Don Neth, MS, for the design and development of the Ektron scanning software, and Christine Kassuba, MA, for editorial assistance.

\section{References}

[1] D.M. Berman and D.W. Russell, Cell-type specific expression of rat steroid 50< reductase isozymes, Proc. Natl. Acad. Sci. 90 (1993), 9359.

[2] B. Chapman, Fixing to switch, CAP Today, August, 1996, 52-62.

[3] M.K. Cohen, D.A. Arber, K.S. Coffield, G.T. Keegan, J. McClintock and V.O. Speights, Jr., Neuroendocrine differentiation in prostatic adenocarcinoma and its relationship to tumor progression, Cancer 74(7) (1994), 1899-1903.

[4] S.S. Epstein, E. Arnold, J. Andrea, W. Bass and Y. Bishop, Detection of chemical mutagens by the dominant lethal assay in the mouse, Toxicol. Appl. Pharmacol. 23(2) (1972), 288-325.

[5] A. Gaddipati, J. Cornhill, E. Herderick and R. Yagel, An efficient method for automated segmentation of histochemically stained slides, in: 17th Annual Conference IEEE-EMBS, Montreal, Canada, 1995.

[6] M. Grist and J. Chakraborty, Identification of a mucin layer in the urinary bladder, Urology 44(1) (1994), 26-33.

[7] J.A. Hunt, D.G. Vince and D.F. Williams, Image analysis in the evaluation of biomaterials, J. Biomed. Engrg. 15(1) (1993), 39-45.

[8] H. Kaneto, J. Morrissey, R. McCracken, A. Reyes and S. Klahr, Enalapril reduces collagen type IV synthesis and expansion of the interstitium in the obstructed rat kidney, Kidney Int. 45(6) (1994), 1637-1647.

[9] W.D. Kerns, K.L. Pavkov, D.J. Donofrio, E.J. Gralla and J.A. Swenberg, Carcinogenicity of formaldehyde in rats and mice after long-term inhalation exposure, Cancer Res. 43(9) (1983), 4382-4392.

[10] S. Krishnamurthy and Y. Dayal, Pancreatic metaplasia in Barrett's esophagus. An immunohistochemical study, Am. J. Surg. Pathol. 19(10) (1995), 1172-1180.

[11] F.S. Leach et al., p53 mutation and Mdm2 amplification in human soft tissue sarcomas, Cancer Res. 53(10) (1993), 2231-2234.

[12] G. MacDonald, P. Dillman and I. Shirley, Evaluation of six histological fixatives using image analysis to measure reaction product concentration, J. Histotechnol. 18 (1995), 119.

[13] R. Noonan (Northview Pacific Laboratories), Acute toxicity testing - Histochoice tissue fixative. 28 May 1993, Report No.: X3B130G, 1996.

[14] Occupational exposure to formaldehyde (29 CFR 1910), in: Federal Register, Vol. 50, 1992, p. 22290.

[15] D. Sheehan and B. Hrapchak, Fixation, in: Theory and Practice of Histotechnology, Mosby, Columbus, 1980, pp. 4058 . 
[16] S.R. Tahan, Y. Wei, P. Ling and B.R. Bistrian, Influence of formalin fixation time and tissue processing method on immunoreactivity of monoclonal antibody PC10 for proliferating cell nuclear antigen, Modern Pathol. 8(2) (1995), $177-182$.

[17] C. Taylor and S. Shi, Fixation, processing, special applications, in: Immunomicroscopy: A Diagnostic Tool for the Surgical Pathologist, C. Taylor and R. Cote, eds, 2nd edn, Vol. 19, W.B. Saunders, Philadelphia, 1994, pp. 42-70.

[18] D. Vince, O. Demirkaya, R. Cothren and J. Cornhill, Quantitative analysis of inflammatory cells in aortic atherosclerosis of young adults, Cardiovasc. Pathol. 5(1) (1996), 3-10.

[19] C.C. Yu, A.L. Woods and D.A. Levison, The assessment of cellular proliferation by immunohistochemistry: a review of currently available methods and their applications, Histochem. J. 24(3) (1992), 121-131. 


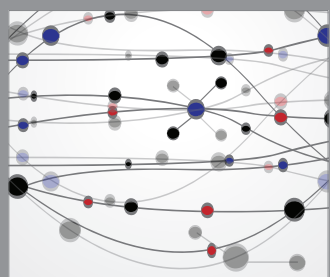

The Scientific World Journal
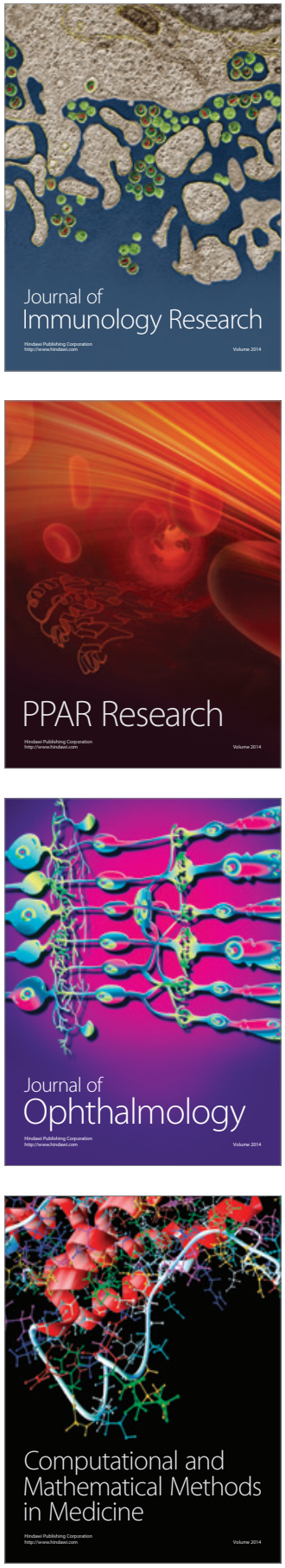

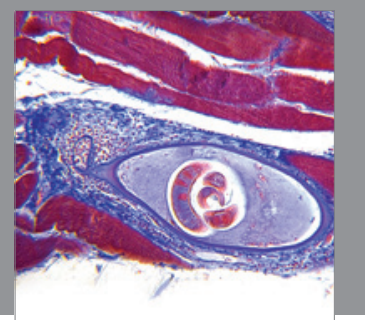

Gastroenterology

Research and Practice
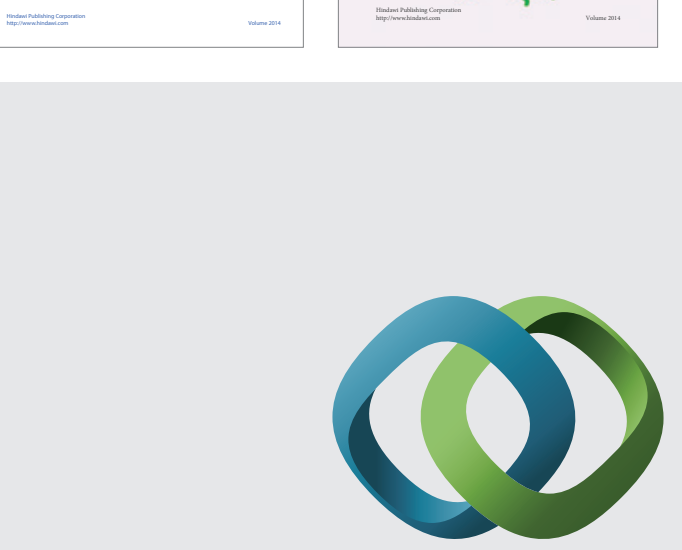

\section{Hindawi}

Submit your manuscripts at

http://www.hindawi.com
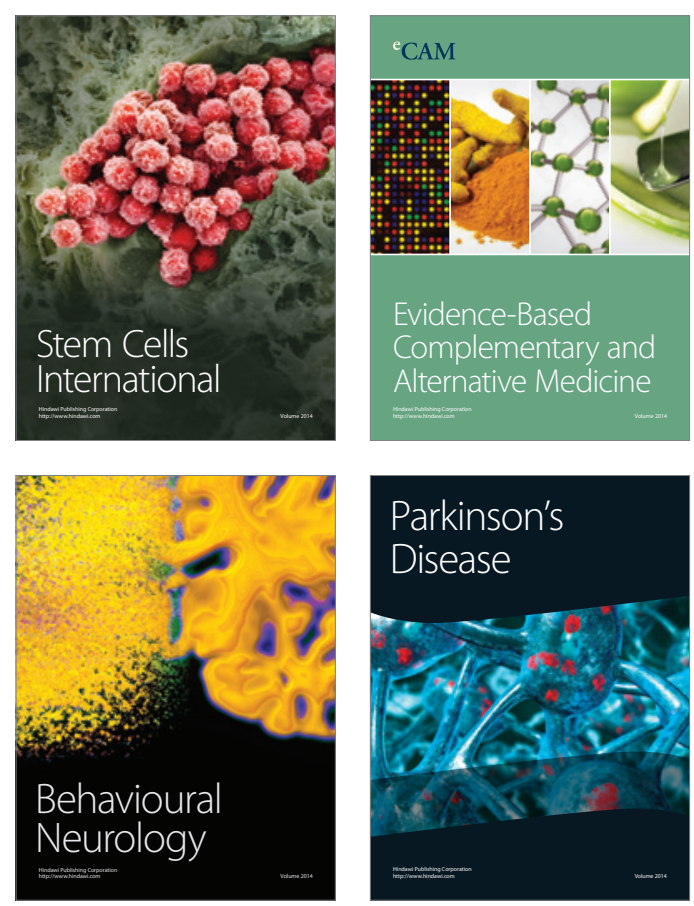

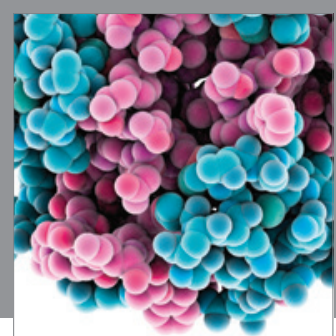

Journal of
Diabetes Research

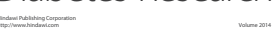

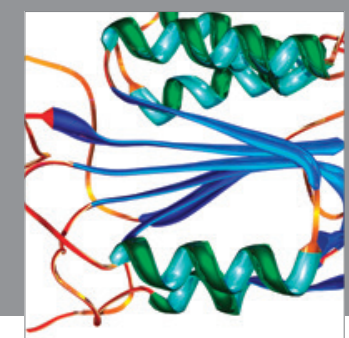

Disease Markers
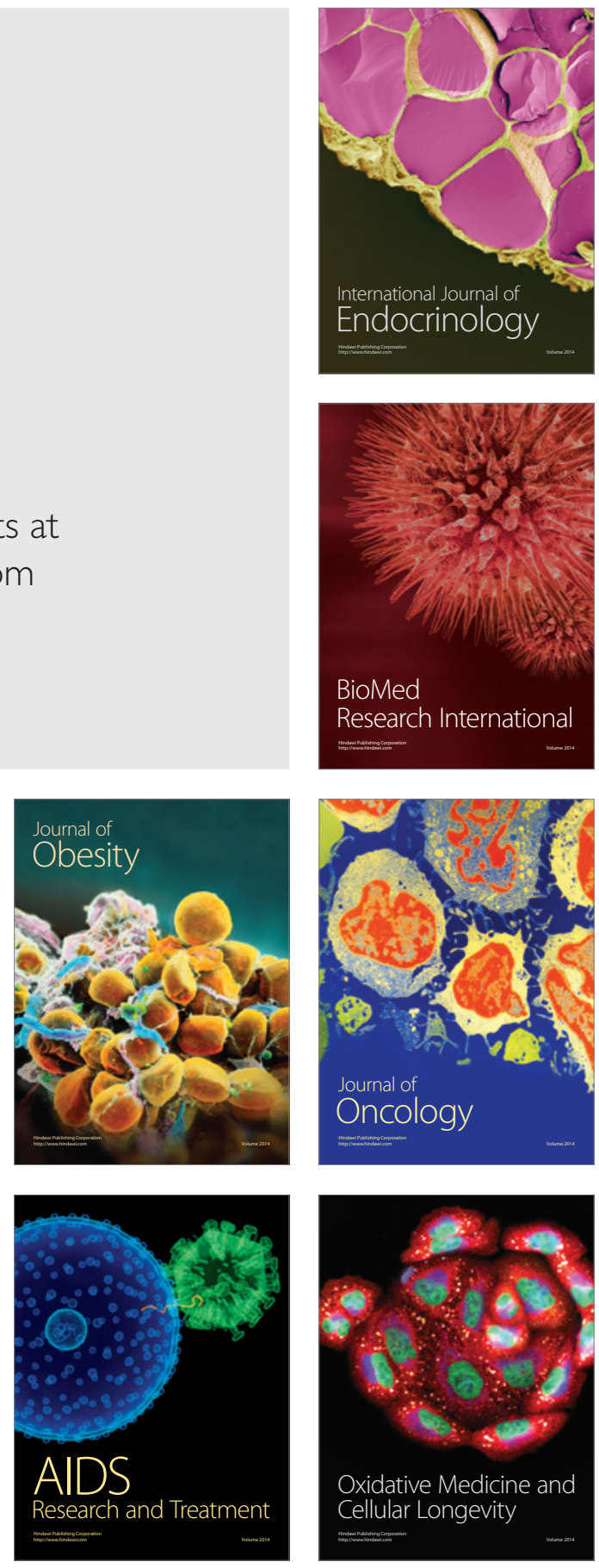